\title{
A Virtual Uneven Grid-Based Routing Protocol for Mobile Sink-Based WSNs in a Smart Home System
}

\author{
Xiaodong Liu ${ }^{1}$ and Qi Liu ${ }^{1}$ \\ ${ }^{1}$ School of Computing, Edinburgh Napier University, 10 Colinton Road, Edinburgh, EH10 \\ 5DT, UK \\ q.liu@napier.ac.uk
}

\begin{abstract}
In a non-uniformly distributed network, the data-concentrating centre equipped with sparse nodes rapidly depletes its battery energy due to the hotspot problem. To solve this problem, a Virtual Uneven Grid-based Routing protocol (VUGR) is proposed in this paper, which aims to prolong the stable network operating time by dynamically partitioning grid cells, so as to handle energy resources into smaller cells to form such uneven grid cells. A maintenance mechanism in a smart home environment for the higher-layer virtual structure is also adopted in the VUGR to ensure the stable operation of the entire network. In addition, latest location information is updated regularly to all regions within the network. Simulation results demonstrate that the VUGR protocol performs better compared to existing solutions.
\end{abstract}

Keywords: Uneven Grid, Sink Mobility, Hotspot Problem, Dynamic Route Adjustment

\section{Introduction}

Typical wireless sensor networks (WSNs) consist of a lot of micro devices, which have the ability to perform many tasks, e.g., environment sensing, data processing and communicating $[1,2]$. Due to these features, smart micro devices are often applied into different domains, especially those dangerous or unattended physical environment, such as battle reconnaissance, medical services, forest fire monitoring, volcanic monitoring, agricultural production and industrial manufacturing [3-5]. However, in these application domains, supplying power for sensor devices is difficult. Therefore, traditional WSNs aim to be designed as energy efficient networks which can maximize the network lifetime [6].

In a multi-hop wireless sensor network, due to data convergecast features in traditional data transmission approaches that result in higher data traffic around the static sink, sensor nodes in the vicinity of the sink will consume more energy in packet forwarding than other nodes in the rural area, as therefore leads to the energy-hole problem [7]. Sink mobility is proposed as a feasible solution to this problem [8-10]. As mobile sinks move to the next sojourning position, the hotspots in the vicinity of the sinks will 
be shifted, which helps acquiring uniform energy dissipation of nodes to extend the network lifetime.

Exploring sink mobility helps to mitigate the hotspot problem, thus balance node energy consumption and prolong network lifetime effectively. However, it also brings new challenges to data dissemination. Unlike static sink scenarios, the network topology becomes dynamic as mobile sinks change their positions continuously [11].

To enable the latest location information of the mobile sink to be easily forwarded to all high-tier nodes, flooding the latest location information of the mobile sink among high-tier nodes is the simplest method. However, communication overhead caused by flooding is huge. So how to keep fresh routes towards the sinks on the move of mobile sinks is a core problem [12]. The approach of overlaying a virtual infrastructure over the physical network has been proposed to enhance the data transmission efficiency as well as decrease node's energy dissipation in the case of mobile sinks $[13,14]$. In such virtual infrastructure-based sensor networks, e.g. a smart home or smart building environment, sensor nodes often play two roles: high-tier nodes and regular nodes. Hightier nodes are responsible for forwarding data packets generated from low-tier regular nodes to the sink. Regular nodes generate data packets on the basis of the physical environment and deliver them to their respective high-tier nodes.

In a smart home environment enabled by the virtual infrastructure, maintaining its complete infrastructure during the period of network operation can significantly affect network performance. Switching the role of high-tier nodes (major cluster headers, partial cluster headers, etc.) with regular nodes during the WSN operation can not only dramatically prolong the network lifetime, but extend the death time of nodes as well.

In this paper, we propose an energy efficient routing protocol for a WSN-based smart home system, called Virtual Uneven Grid-based Routing (VUGR). The VUGR divides the WSN network into several uneven grids, aiming to avoid formation of the energy hole due to non-uniform distribution of sensor nodes. A sensory data collection strategy with a single mobile sink is developed to collect data from the network in the VUGR protocol.

The remainder of this paper is organized as follows. Section 2 reviews several virtual infrastructure-based routing protocols. In Section 3, the VUGR protocol is discussed in detail, including the virtual uneven grid construction approach and mobile sink-based sensory data collection scheme. Section 4 evaluates the proposed VUGR protocol. Finally, the paper is concluded in section 5 .

\section{Related Work}

In a mobile-sink based wireless sensor network, the network topology becomes dynamic as mobile sinks move. To cope with the dynamic network topology, overlaying a virtual infrastructure over the physical network is a feasible solution. In recent years, researchers have already proposed many virtual infrastructure-based protocols, intending to find an energy-efficient virtual high-layer structure and communication mechanism to improve network performance. The popular network topologies include grid- 
based network topology, area-based network topology and cluster-based network topology, etc.

A cluster-based structure is the most popular network topology, which attracts lots of researchers to focus on it. A three-layer framework for mobile data collection includes the sensor layer used for generating data packets about monitoring objects, cluster head layer used for transferring data packets from the sensor layer, and mobile collector layer used for gathering data packets from the network. The cluster head layer constitutes the virtual infrastructure of the network. Then, a distributed load balanced clustering and dual data uploading (LBC-DDU) [15] based on the three-layer framework is proposed. The LBC-DDU employs multiple cluster heads in each cluster. Cluster heads in each cluster collaborate to select a sojourning position for the mobile sink and then send the sojourning location information to the mobile sink. The mobile sink staying on the edge of the network adopts a shortest path algorithm to find an optimized moving path from several sojourning points. Even though the LBC-DDU can greatly reduce energy consumption by alleviating routing burdens on nodes, the higher communication delay is inevitable for the LBC-DDU.

In a grid-based network, each node only needs to maintain a simple forwarding node set to transmit data, and so this type of network has more efficient performance in data transmission. Two-Tier Data Dissemination (TTDD) is a scalable and efficient gridbased data dissemination approach that enables multiple mobile sinks to continuously receive data on the move [16]. The TTDD limits flooding queries into a local grid cell, thus decreasing the overall energy dissipation. However, frequent grid construction overhead cannot be ignored, which makes it not suitable for periodic data reporting application since it needs to build a grid for all sources along the sink's trail.

Virtual Grid-Based Dynamic Routes Adjustment Scheme (VGDRA) is a mobilesink based algorithm [13], which only constructs one grid structure. Cell-headers elected in each grid cell re-adjust their routes dynamically to maintain the latest location information of the mobile sink, thereby minimizing the communication cost. The VGDRA shows good performance in alleviating the hotspot problem; however, in a non-uniformly distributed network, the uniform gird construction approach causes different node densities in each grid cell. Grid cells with sparse nodes along the sink's moving trajectory can prematurely deplete all the node energy, and therefore lead to the energy-hole problem.

To alleviate the hotspot problem, Prince et al. proposed an Energy Efficient Uneven Grid Clustering based Routing protocol (EEUGCR) [17], which divides the whole network into several rectangular grids of unequal size via the base station in a centralized approach. The EEUGCR considers the distance between source nodes and the base station; however, in a non-uniformly distributed network, this method may bring worse results if nodes around the base station is sparse. The EEUGCR is limited by data collection methods.

A cluster-based network structure attracts the most researchers due to easy deployment and low complexity. Moreover, the VGDRA and EEUGCR also adopt the idea of clustering. Member nodes in each grid only need to know the routing information to cell-headers and send the sensing data from the physical environment to cell-headers. 
Cell-headers only need to forward packets from low-tier nodes to the next hop based on the routing table, which stores the routing information between cell-headers.

Besides the above two types of network structures, the area-based network topology is also well investigated, such as LBDD (Line-Based Data Dissemination) [18], VLDD (Virtual Line-Based Data Dissemination) [19] and Ring Routing [11].

The LBDD [18] defined a vertical virtual line, where in-line nodes becomes hightier nodes. Source nodes forward data packets to in-line nodes and these data packets are stored in the closest in-line nodes. Mobile sinks send a query within the line area. In-line nodes upon receiving this request message share it within the line area until this request message reaches in-line nodes storing target data packets. This in-line node sends data packets to mobile sinks directly. However, the LBDD suffers serious hotspot problem, especially for large-scale networks. To alleviate it, it is necessary to build a wide enough line area for the network since sharing request messages from mobile sinks within the line area will cause huge communication overhead.

Ring Routing [11] is a ring-based routing protocol, which constructs a circular ringbased high-layer network structure. Ring nodes are used for storing latest location information of mobile sinks. Regular nodes acquire locations of mobile sinks by retrieving ring nodes, and then send generated data packets to mobile sinks directly. To reduce communication overhead between regular nodes and ring nodes, regular nodes only query ring nodes for latest location information of mobile sinks after a certain interval, and so location information of mobile sinks may be out of date when regular nodes perform the data delivery process. The Ring Routing protocol employs a follow-up mechanism to help these data packets with wrong destinations to complete data delivery. When mobile sinks change their positions, they will set the closest neighbouring nodes as anchors and broadcast their role of anchor nodes within their communication range. Data packets from regular nodes will be transferred to anchor nodes. When mobile sinks will leave their communication range, they will select new anchors and inform the role of new anchors to old anchors. Old anchors upon receiving data packets from source nodes will forward them to next new anchors until these packets arrived at mobile sinks. The ring structure alleviates the hotspot problem to a certain extent. However, ring routing may lead to higher communication overhead in a large-scale network due to a lot of data request between regular nodes and ring nodes.

Other types of network topologies include Railroad (railroad-based network topology) [20], QDD (tree-based network topology) [21], and HCDD (honeycomb-based network topology) [22] etc.

In general, a successful virtual infrastructure-based routing protocol has an easily implemented hierarchical structure, which enables high-tier nodes (i.e. cluster headers) to be aware of the latest routes towards the sink so other nodes to acquire the latest position of high-tier nodes from the high-tier virtual infrastructure. However, the nodes are randomly distributed in an inhomogeneous network and the parts of different nodes in the network have different density. Uniformly dividing the grid, such as the VGDRA, will lead to the imbalance of resources in each cluster, and the large difference of the survival time of each cluster will even result in "energy hole". A Virtual Uneven GridBased Routing protocol (VUGR) is therefore proposed in this paper to solve the problem, where it divides the poor-resource grid into multiple virtual sub-clusters according 
to the running-time network state. A sub-cluster unit with poor resource status does not participate in the election of upper-level headers and is therefore no longer included in the sink node's moving path, which will greatly save the energy of nodes in the subcluster.

\section{$3 \quad$ Algorithm Design}

In this section, a Virtual Uneven Grid-Based Routing protocol (VUGR) is presented and discussed in detail. The VUGR partitions grid cells at low energy levels into smaller cells, which seek grid cells at high energy levels to forward data packets without participating in construction of virtual high-layer structures. In the VUGR protocol, sensor nodes within the network are assigned three roles, as shown in Fig. 1; they are regular nodes (RNs), main cell-headers (MCHs), and assistant cell-headers (ACHs). The $\mathrm{MCHs}$ form a virtual high-layer structure aiming at following three objectives:

- How to construct a virtual uneven grid structure;

- How to send data from RNs to mobile sinks;

- How to maintain a high-layer structure.

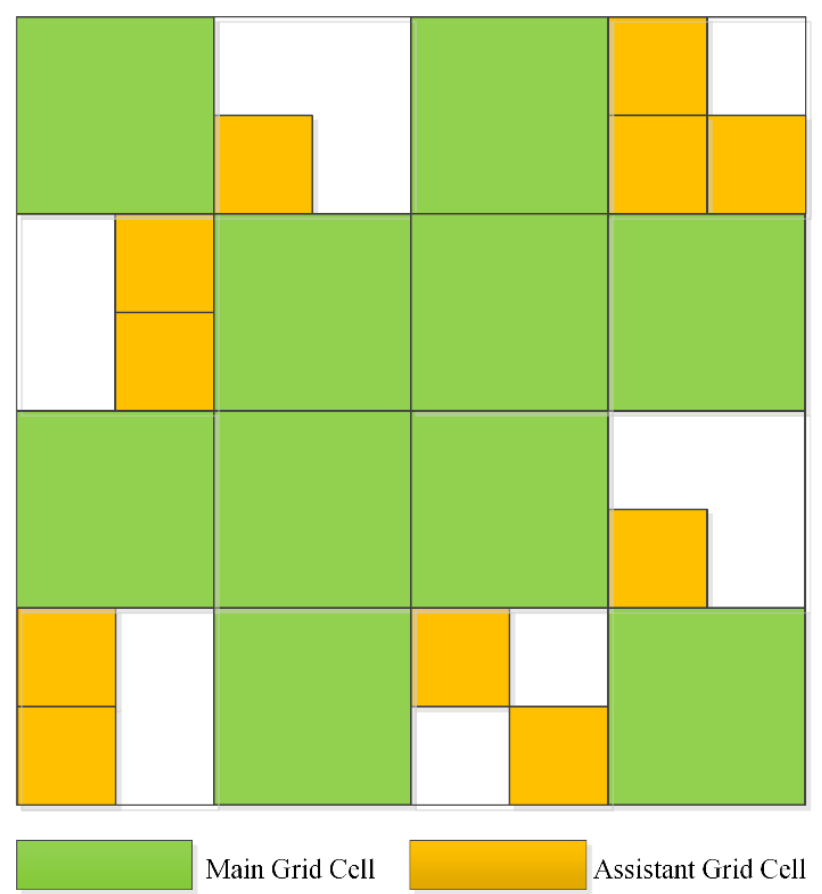

Fig. 1. A Typical Structure of a Virtual Uneven Grid

\subsection{Assumptions of a VUGR-enabled Network}

Following assumptions have be made on the VUGR: 
- $\quad$ All sensor nodes in the sensor field are randomly distributed and then keep static after deployed successfully.

- All sensor nodes have the same battery capacity; however, mobile sinks have no energy constraints.

- No obstacles in the external environment hinder communications between nodes.

- Fundamental communication and relevant processes of all sensor nodes follow the general design specified by the VGDRA [13] and Ring Routing [11].

- All sensor nodes are aware of their location information which can be easily achieved by a positioning system or an energy-efficient positioning algorithm.

\subsection{Construction of a Virtual Uneven Grid}

To extend stable operation time of WSN, the VUGR dynamically adjusts grid cells with low average energy levels to achieve uniform energy consumption among grid cells. Entire processes can be divided into two stages, i.e. a Normal Operation phase and a Dynamic Adjustment phase.

\subsubsection{A Normal Operation Phase}

Originally, the VUGR divides the deployment area into several uniform grids with equal size. The number of grid cells is determined by the total number of sensor nodes in the deployment area. 5\% of the total number of sensor nodes is considered as the approximate optimal number of grid cells [23]. Assuming $K$ is the number of grid cells and $N$ is the total number of sensor nodes. Equation (1) is adopted to divide the sensor field into uniform grid cells.

$$
K=\left\{\begin{array}{cc}
4 & N \times 0.05 \leq 6 \\
9 & 6<N \times 0.05 \leq 12 \\
16 & 12<N \times 0.05 \leq 20 \\
\vdots & \vdots
\end{array}\right.
$$

After completing the network partition, every sensor node belongs to a single grid cell. Based on their location information which is expressed as coordinates $(x, y)$, every sensor node can know their serial number of grid cells according to equation (2), where $K$ represents the number of grid cells, and $L$ is the edge length of the entire area.

$$
\text { Cell }_{x}=\left\lceil\frac{x}{\frac{L}{\sqrt{K}}}\right\rceil=\left\lceil\frac{x \sqrt{K}}{L}\right\rceil, \text { Cell }_{y}=\left\lceil\frac{y}{\frac{L}{\sqrt{K}}}\right\rceil=\left\lceil\frac{y \sqrt{K}}{L}\right\rceil
$$

Furthermore, equation (3) is used to calculate coordinates of mid-point of each cell. Sensor nodes' distances to mid-point of cells can be easily achieved using the Euclidean distance.

$$
\operatorname{Mid}_{x}=\frac{L}{\sqrt{K}}\left(\operatorname{Cell}_{x}-\frac{1}{2}\right), \operatorname{Mid}_{y}=\frac{L}{\sqrt{K}}\left(\operatorname{Cell}_{y}-\frac{1}{2}\right)
$$

Nodes whose distances to mid-point of cells are closest will be elected as first-round cell-headers, which form the network backbone called MCHs. As shown in Fig. 2, a mobile sink is defined to obtain data from the environment. 


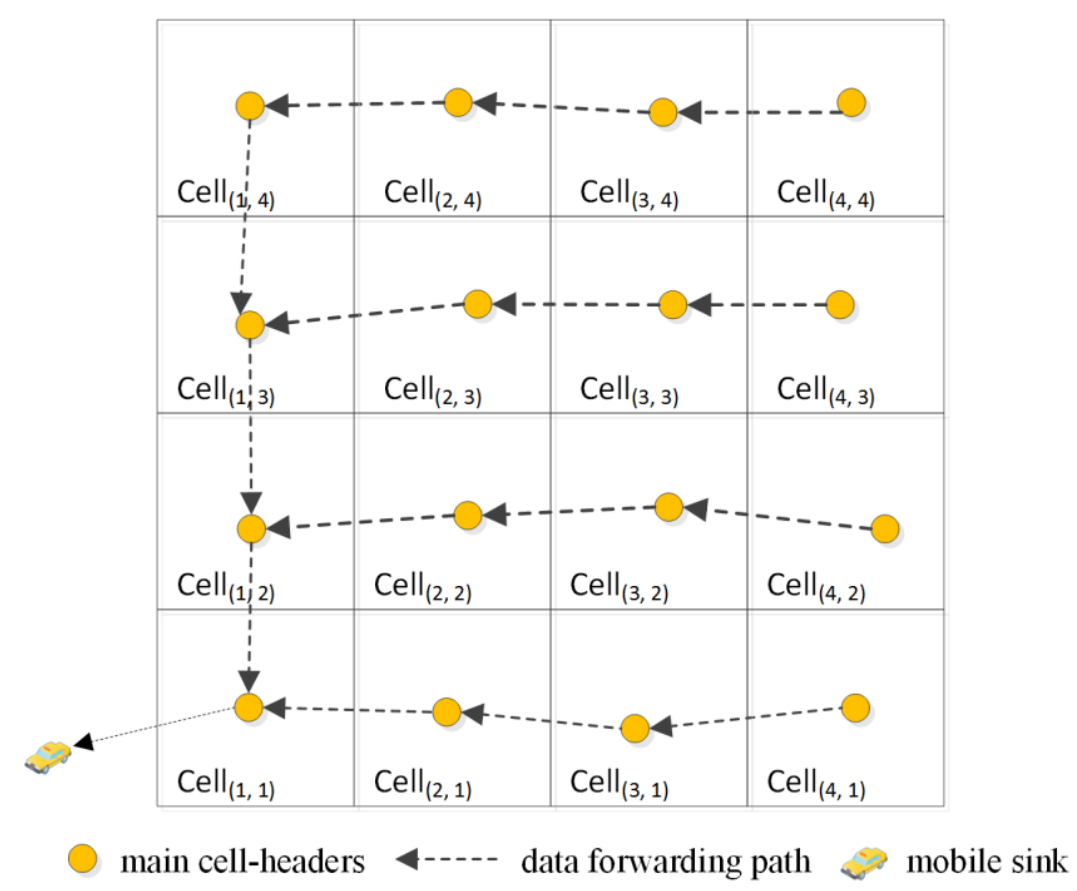

Fig. 2. Data Concentrating Process in Normal Operation Phase

\subsubsection{A Dynamic Adjustment Phase}

In the Normal Operation phase of the WSN, a mobile sink moves counter-clockwise around the network edge to collect data packets, which can be seen in Fig. 2. Cell $(1,1)$ becomes the data concentrating centre and so the overall energy consumption of Cell $(1,1)$ increases significantly. When the mobile sink changes its sojourning position, grid cells around the network edge will becomes the data concentrating centre one by one, i.e. $\operatorname{Cell}_{(2,1)}, \operatorname{Cell}_{(3,1)}, \operatorname{Cell}_{(4,1)}, \operatorname{Cell}_{(1,2)}, \operatorname{Cell}_{(4,3)}, \operatorname{Cell}_{(4,4)}, \operatorname{Cell}_{(3,4)}, \operatorname{Cell}_{(2,4)}, \operatorname{Cell}_{(1,4)}, \operatorname{Cell}_{(1,3)}$ and Cell $_{(1,2)}$ respectively. If these grid cells own hard energy supplying, it is highly possible that part of grid cells will fail at the beginning of the network operation, which may have a great impact on normal network operation. As a result, WSN should have corresponding strategies to copy with it.

In the Dynamic Adjustment phase, if the current MCHs cannot find the next round of cell-headers, it means that this grid cell own hard energy resources and cannot participate in construction of virtual high-layer structures. Using the same method described in the Normal Operation phase, these grid cells with hard energy resources are partitioned into several smaller grid cells. Nodes that are closest to the mid-point of cells are elected as cell-headers called ACHs. Fig. 3 is the network backbone after a Dynamic Adjustment phase. 


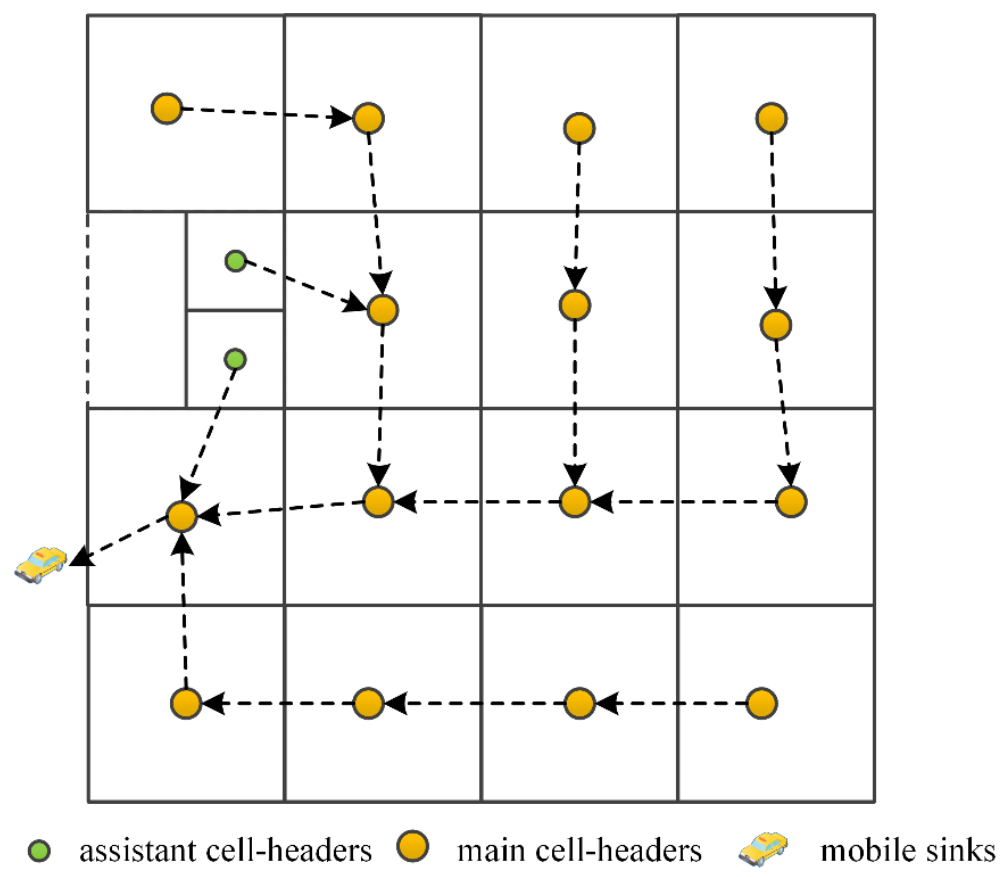

Fig. 3. Data Concentrating Process in Dynamic Adjustment Phase

\subsection{A Mobile-Sink based Sensory Data Collection Scheme}

A dynamic routes adjustment scheme is employed in the Normal Operation phase to facilitate dynamic adjustment of the network topology, which consists of following three aspects.

\subsubsection{Data Forwarding Processes between ACHs and MCHs}

Nodes from assistant grid cells are only responsible to acquire information from the physical environment and then forward it to its adjacent $\mathrm{MCH}$. The process of the data forward from an $\mathrm{ACH}$ to its host $\mathrm{MCH}$ is shown below. As depicted in Fig. 4, the ACHs select their adjacent MCHs as their next-hop.

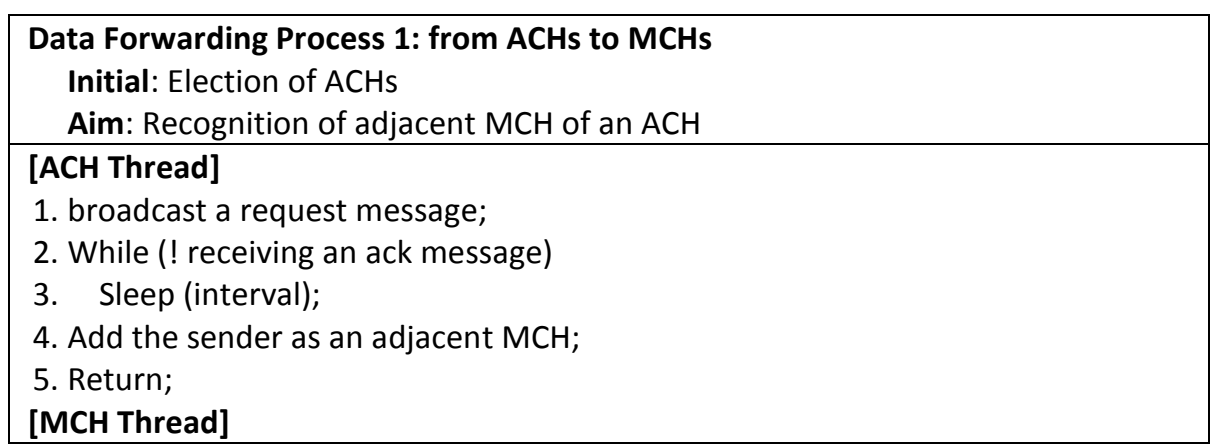


1. If a request message is received, then

2. Sends an acknowledge message;

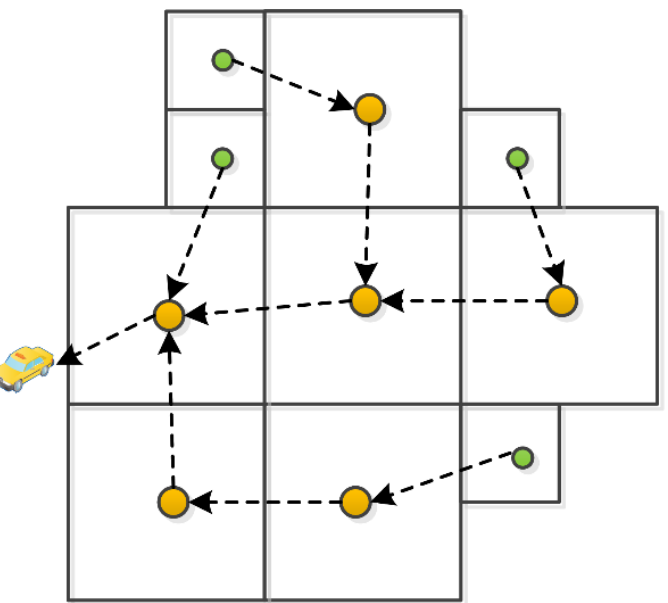

○ assistant cell-headers $\bigcirc$ main cell-headers

mobile sinks

Fig. 4. Data Forwarding Process between $\mathrm{ACHs}$ and $\mathrm{MCHs}$

\subsubsection{Data Forwarding Processes between MCHs}

Grid cells with hard energy resources are partitioned into smaller grid cells and no longer help constructing the virtual high-layer structure. As a result, those MCHs whose next-hop are grid cells need to be dynamically adjusted and have to choose their nexthop again. The specific processes are as follows:

Data Forwarding Process 2: between MCHs

Initial: Downgrade of an $\mathrm{MCH}$ into $\mathrm{ACH}$

Aim: Recognition of a next-hop $\mathrm{MCH}$

Step 1: An MCH informs its member nodes that this grid cell will be partitioned again and shares this message with adjacent $\mathrm{MCH}$ at the same time;

Step 2: An adjacent $\mathrm{MCH}$ receiving this message will delete the sender from its neighbour node table and check whether its next-hop is the sender or not. If the next-hop entry of the adjacent $\mathrm{MCH}$ is the sender, it executes Step 3;

Step 3: The adjacent $\mathrm{MCH}$ employs a greedy algorithm to select their next-hop from its neighbour node table, where a node with the minimum distance to the mobile sink will be selected as the next-hop.

In addition, to avoid as an MCH. Traditional election of a cluster header may suffer from the problem that a particular node is elected due to its higher energy level and better position to the mobile sink. Downgrading an $\mathrm{MCH}$ into an $\mathrm{ACH}$ helps avoid such the problem when average energy level of the grid is low. 
To better describe data forwarding processes between MCHs, every cell is given a unique serial number. As shown in Fig. 5, Cell $(1,3)$ needs to be further partitioned into smaller grid cells, whereas $\operatorname{Cell}_{(1,4)}$ need to adjust its next-hop from $\operatorname{Cell}_{(1,3)}$ to $\operatorname{Cell}_{(2,4) \text {. }}$

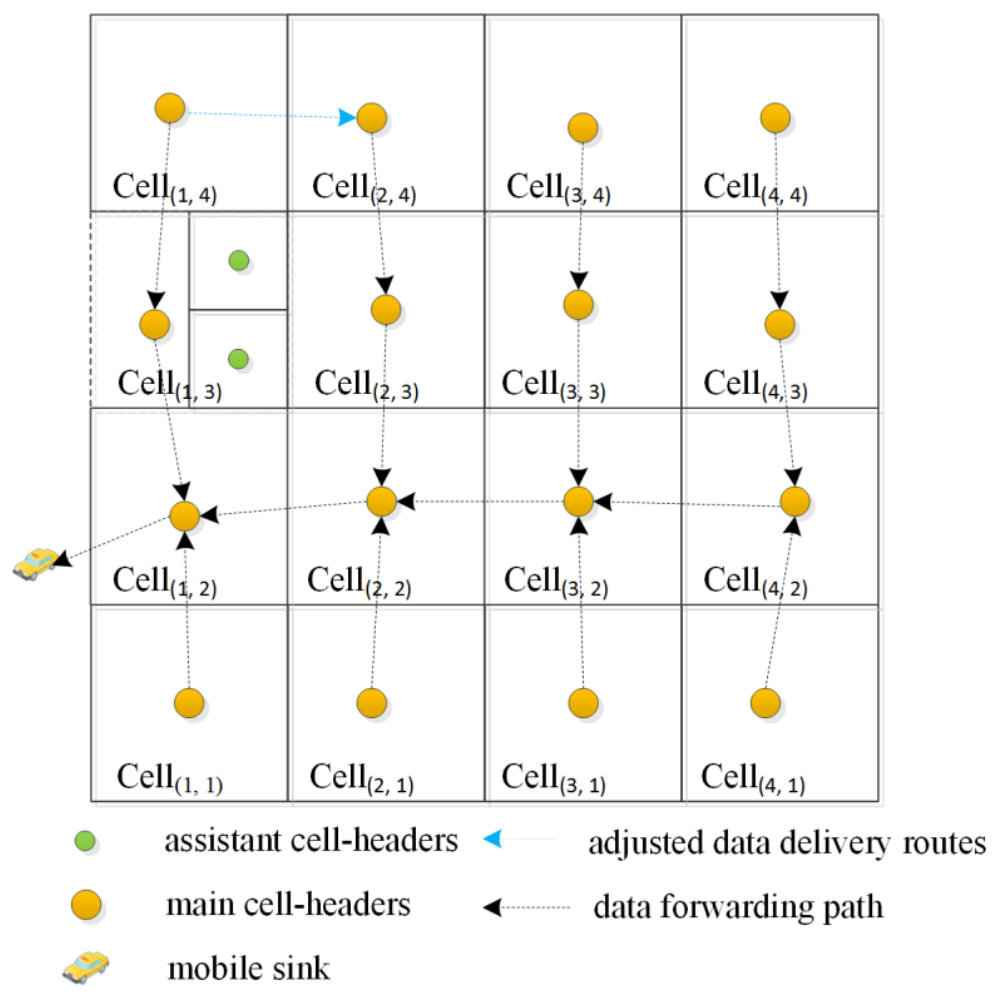

Fig. 5. Data Forwarding Process between MCHs

\subsubsection{A Dynamic Routes Adjustment Scheme}

During the running period of the network, if MCHs cannot find the next hop of cellheaders, then sensor nodes in this grid cell cannot keep monitoring the physical environment. Only main grid cells around the network edge can trigger the dynamic adjustment processes.

When a MCH triggers the dynamic adjustment of grid cells, it will send a message to inform the mobile sink. The mobile sink upon receiving this message will delete this grid cell from its sojourning position and change its moving path. Fig. 6 is an example of adjusting the moving path of the mobile sink. After completing the dynamic adjustment process of grid cells, the mobile sink collects data along the new moving path. MCHs within the network follow the processes below to facilitate the adjustment of the dynamic network topology.

Data Forwarding Process 3: Dynamic Routes Adjustment

Initial: An MCH loses Next-Hop Communication 
Aim: Routes Adjustment

Step 1: The mobile sink shares a location update packet with its adjacent sensor nodes in its communication range. Nodes upon receiving location update packets verify whether the mobile sink is within their communication range or not. If so, nodes adjust their data delivery routes towards the mobile sink and directly communicate with the mobile sink. If not, execute Step 2;

Step 2: Nodes upon receiving location update packets from the mobile sink forward packets to their cell-header. If the node is the originating cellheader, the mobile sink will be informed to communicate directly. Otherwise, execute Step 3;

Step 3: The node upon receiving location update packets becomes the new originating cell-header and shares this update packet with all adjacent cellheaders at the same time. The adjacent cell-headers upon receiving location update packets from the mobile sink check whether their nexthop is the sender or not. If not, these cell-headers set their next-hop as the current originating cell-header and propagate this location update packet to downstream cell-headers. Otherwise, discard location update packets;

Step 4: If the downstream cell-headers do not exist, the location update packet will be discarded. Otherwise, the receiver verifies whether its next-hop is the sender or not. If not, the receiver sets its next-hop as the sender and continues to transmit the update packet to the downstream cell-headers. Repeat to execute this step until completing routing reconfiguration.

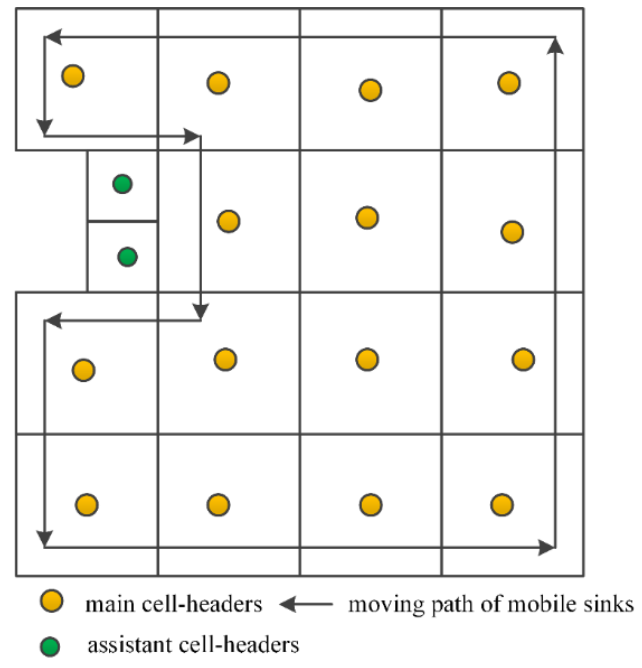

Fig. 6. The Moving Path of Mobile Sinks 
Fig. 7 illustrates the dynamic routes adjustment scheme, where the mobile sink moves from $\operatorname{Cell}_{(2,3)}$ to $\mathrm{Cell}_{(2,2)}$. Step 3 is therefore used to update data delivery routes of $\mathrm{Cell}_{(2,3)}$ and $\mathrm{Cell}_{(3,2)}$. After that, Step 4 is executed to update data forwarding routes towards the mobile sink of Cell $(4,2)$, so as to complete the adjustment of the global network topology.

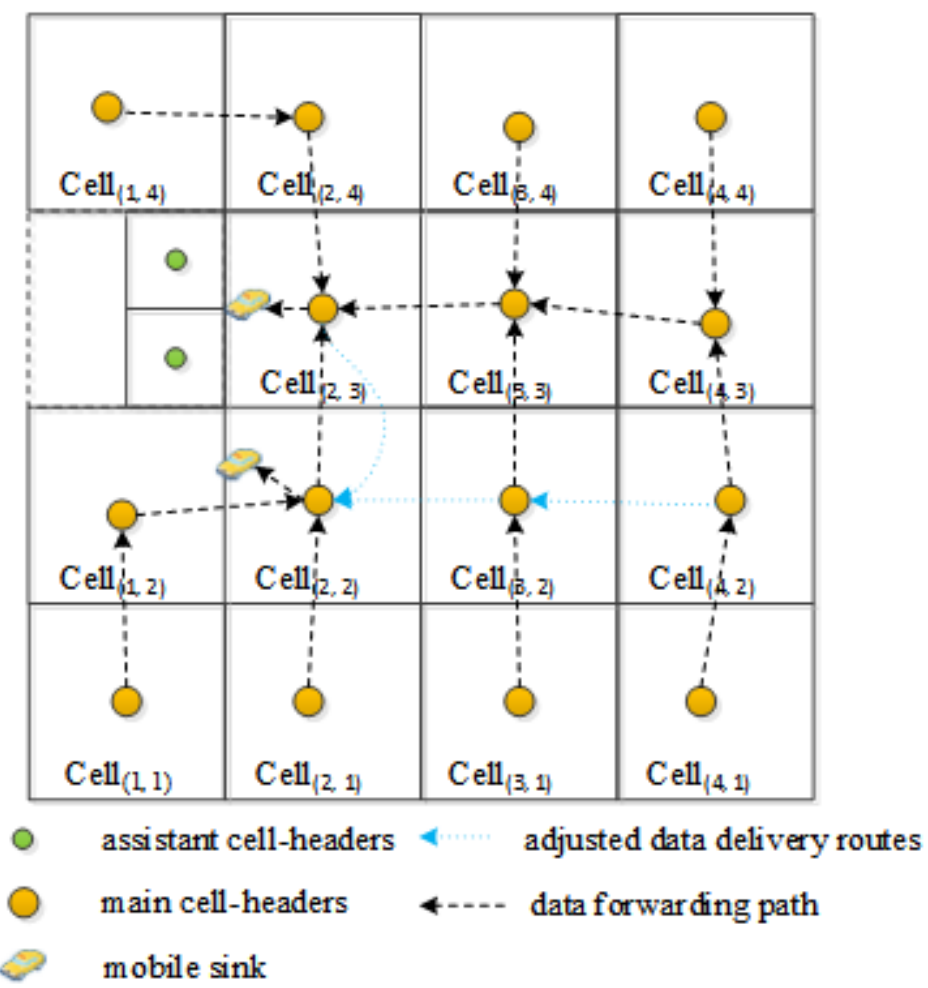

Fig. 7. Dynamic Routes Adjustment Scheme

\subsection{Re-election of Cell-Headers}

Data delivery from member nodes to their corresponding cell-headers is a static data concentrating process. Cell-headers act as a fixed data collector of the local area, which consumes their battery energy faster than other nodes. To ensure that cell-headers of grid cells have enough energy resources to handle huge data traffic, it is necessary to switch the role of cell-header of every cell in the proposed VUGR algorithm. The VUGR employs an energy threshold to trigger the cell-header re-election process. When the current energy of a cell-header is below $20 \%$, it will broadcast a message to inform member nodes to find a set of cell-header candidates and then select the next cell-header.

The VUGR considers the impact of node density, which implies the number of nodes being approximated. If the cell-header is located in the area with high density, it is 
obvious that more member nodes have shorter distance to their cell-headers. Such cellheader distribution also increases the number of nodes that communicate with the mobile sink directly when the mobile sink sojourns beside the cell-headers for data delivery. In the cell-header re-election process, the node that is relatively closer to the midpoint of grid cells, has more neighbouring nodes and a higher energy level compared to other cell-header candidates is elected as the new cell-header. In order to protect high-layer structure of the network, the current cell-header will share the information of the new cell-header with its member nodes and adjacent cell-headers. Moreover, nodes that have already served as the role of cell-headers no longer participate in the next cell-header re-election.

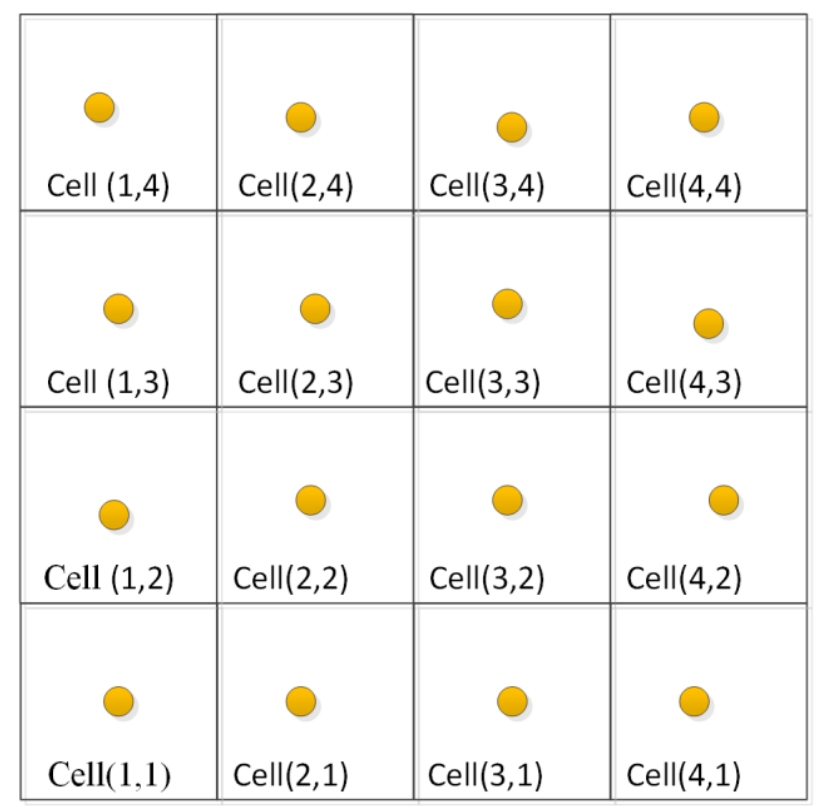

Fig. 8. Grid Cell Distribution

If no suitable node can be found in the search zone, one of two scenarios can occur:

Scenario1: If the grid cell is located at the edge of the senor field, i.e. Cell $(1,1)$, $\operatorname{Cell}_{(2,1)}, \operatorname{Cell}_{(3,1)}, \operatorname{Cell}_{(4,1)}, \operatorname{Cell}_{(4,2)}, \operatorname{Cell}_{(3,3)}, \operatorname{Cell}_{(3,4)}, \operatorname{Cell}_{(2,4)}, \operatorname{Cell}_{(1,4)}, \operatorname{Cell}_{(4,3)}, \operatorname{Cell}_{(1,3)}$ and Cell $_{(1,2)}$ depicted in Fig. 8, the dynamic adjustment process of grid cells will be trigger.

Scenario2: If the grid cell is located inside the sensor field, e.g. Cell $(2,2), \operatorname{Cell}_{(3,2)}$, Cell $_{(1,3)}$ and Cell $_{(2,3)}$, the thresholds being a cell-header candidate will be decreased. 


\section{Performance Evaluation}

\subsection{Simulation Environment}

NS-2.34 is used to evaluate the performance of our proposed VUGR protocol in Ubuntu 10.10. $N$ sensor nodes are randomly deployed in a region of $200 * 200 \mathrm{~m}^{2}$. The simulation area covers a residential community that contains multiple homes. Each cluster ideally assumes a home respectively. After completing the virtual uneven grid construction, the mobile sink moves at a constant speed around the network edge and broadcasts location update packets periodically. The default two-ray-ground propagation model is employed, as well as the default energy model with $d$ as the path loss, which can be calculated using the following formulas, where $K$ is the length of data packets.

$$
\begin{aligned}
& T_{x}=\left(E_{\text {elec }} \times K\right)+\left(\varepsilon_{m p} \times K \times d^{2}\right) \\
& R_{x}=E_{\text {elec }} \times K
\end{aligned}
$$

Part of simulation parameters are listed in Table 1.

Table 1. Simulation Parameters

\begin{tabular}{ll}
\hline \multicolumn{1}{c}{ Parameters } & \multicolumn{1}{c}{ Value } \\
\hline Simulation Area & $200 \times 200 \mathrm{~m}^{2}$ \\
Number of Nodes & $100,150,200,250,300$ \\
Packet Size & 512 bytes \\
Eelec & $50 \mathrm{~nJ} / \mathrm{bit}$ \\
$\varepsilon_{\mathrm{mp}}$ & $10 \mathrm{~nJ} / \mathrm{bit} / \mathrm{m}^{2}$ \\
Einit $_{\text {ink }}$ & $2 \mathrm{~J}$ \\
Esink $_{\text {Simulation Time }}$ & $\infty 2000 \mathrm{~s}$ \\
MAC & IEEE 802.15 .4 \\
V & $5 \mathrm{~m} / \mathrm{s}$ \\
\hline
\end{tabular}

\subsection{Results Analysis}

The performance of the VUGR protocol has been evaluated compared with VGDRA and Ring Routing in terms of Network Lifetime, Data Delivery Ratio and End-to-End Delay.

\subsubsection{Network Lifetime}


Network Lifetime is defined as the death time for the first node. This subsection discusses the performance of VUGR in terms of network lifetime. The maximum number of sensor nodes is 300, so the deployment area should be partitioned into 16 grid cells of same size. As depicted in Fig. 9, the Network Lifetime of three algorithms in different speeds of the mobile sink is calculated and compared. The VUGR, VGDRA and Ring Routing have chosen effective solutions to dynamic network topology, which transmits latest location information of the mobile sink to high-tier nodes. All three algorithms show good convergence as the speed of the mobile sink increases.

Meanwhile, the proposed VUGR algorithm maintains longer network lifetime that the other two when the sink is moving. This is mainly because it considers unbalanced energy resources in every cell when sensor nodes are randomly deployed, therefore partitions grid cells with low energy resources into several smaller grid cells. After that, these cells are not allowed to appear at the moving path of the mobile sink, which avoid further energy consumption as data centres.

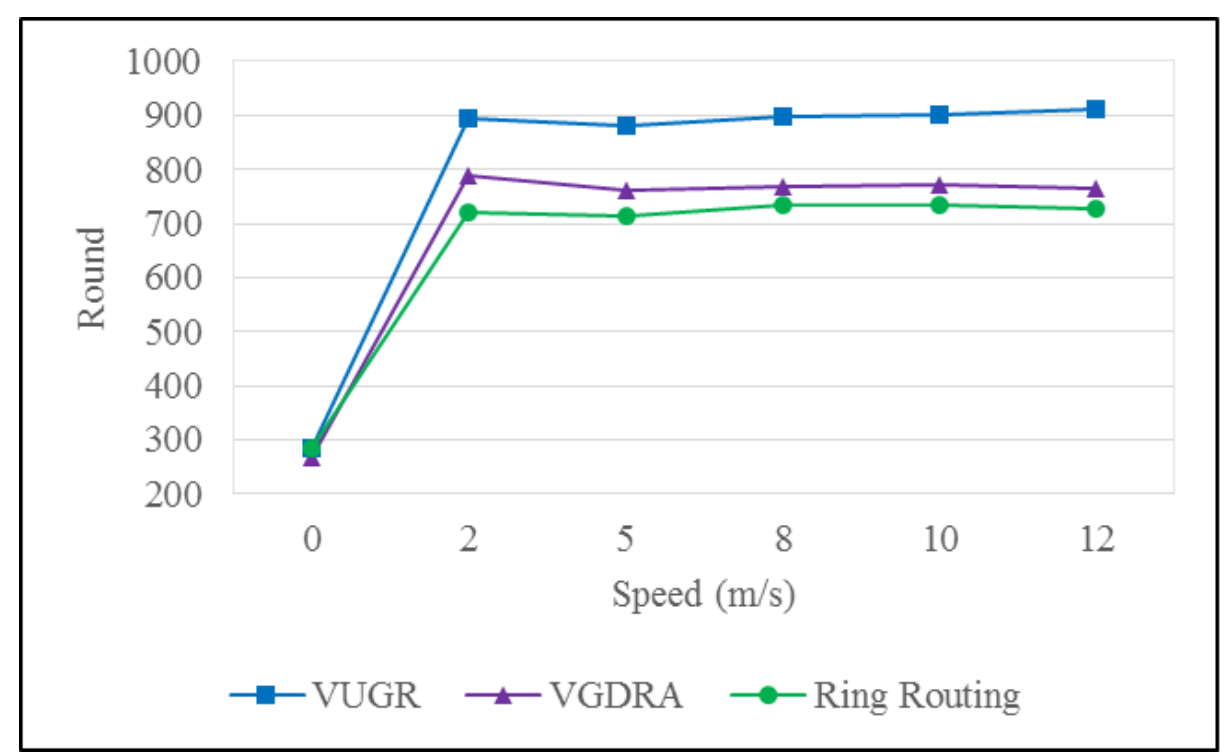

Fig. 9. Network Lifetime in Different Speeds of the Mobile Sink

With different network sizes, the VUGR also shows better network lifetime than the others, as depicted in Fig. 10, where the speed of the mobile sink is constant at $10 \mathrm{~m} / \mathrm{s}$. The Ring Routing neglects the impact of node distribution on the network structure. As the network size increases, sensor nodes near the ring node suffer the hot-spot problem. The VUGR removes grid cells with low energy resources from data concentrating centre permanently, so significantly alleviates the hot-spot problem. 


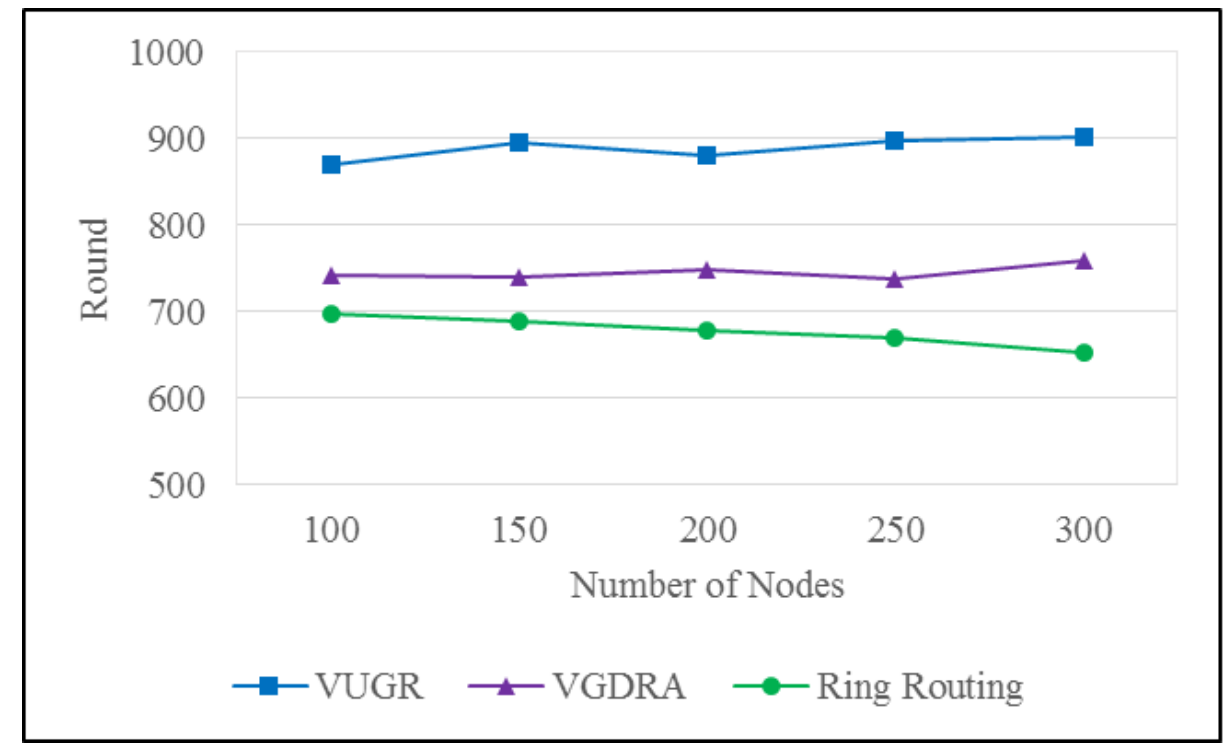

Fig. 10. Network Lifetime in Different Network Sizes

\subsubsection{Data Delivery Ratio}

Fig. 11 shows Data Delivery Ratio (DDR) of three algorithms, where the speed of the mobile sink is at $10 \mathrm{~m} / \mathrm{s}$ and the total number of sensor nodes is 300 . After 926 rounds of data reporting, the DDR of the VGDRA starts to decrease sharply since the virtual uneven grid structure is destroyed, due to the fact that failure of the local network structure hinders data delivery. The proposed VUGR algorithm improves the problem by dynamically adjusting grid cells, which extends the network lifetime. The Ring Routing has the best DDR among three algorithms since it is difficult to destroy the ring structure. Regular nodes can acquire location information of the mobile sink from ring nodes as long as the ring structure exists. Moreover, the Ring Routing employs an anchor-following scheme to help forwarding data packets. Those Nodes which cannot get the latest location information of the mobile sink due to communication delay can complete data delivery towards the mobile sink by surrounded anchors.

Although the VUGR does not achieve the best performance on the DDR, it has secured the virtual high-layer structure for longer time than the VGDRA. 


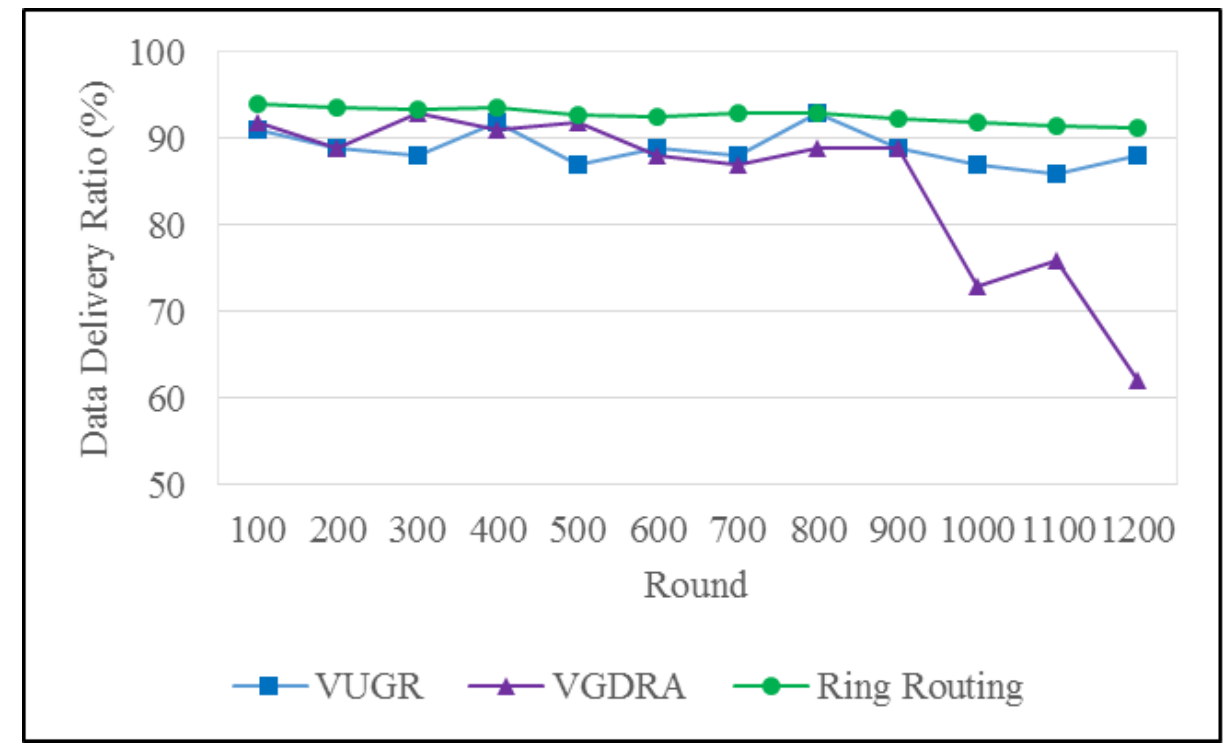

Fig. 11. Data Delivery Ratio

\subsubsection{End-To-End Delay}

This End-to-End Delay (EED) in the experiment is the average delay within the network lifetime.

In Fig. 12, the proposed VUGR algorithm has demonstrated the best performance on the EED among three algorithms as the speed of the mobile sink increases. The total number of sensor nodes is 300. The VUGR and VGDRA have both employed a dynamic routes adjustment scheme to facilitate fast network convergence, therefore leads to lower EED. In addition, the VUGR improves the problem of unbalanced energy resources by changing the high-layer network structure, which always makes the data concentrating centre match to the network centre. Hence, the VUGR has depicted lower communication delay than the VGDRA. The Ring Routing, however, does not support real-time updates of location information within the network. To send data packets to the mobile sink accurately, the Ring Routing employs an anchor-following scheme to help source nodes to complete data delivery. Accordingly, long-distance multi-hop data forwarding has caused higher communication delay. 


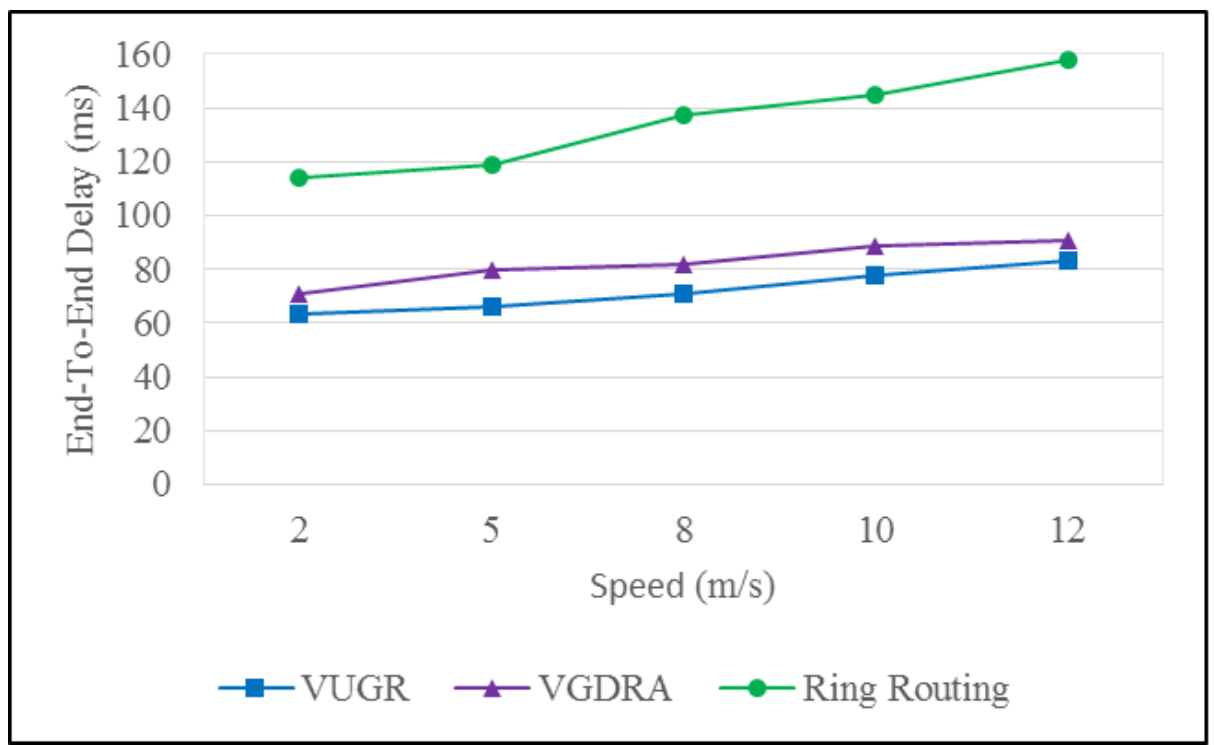

Fig. 12. End-to-end Delay in Different Speeds of the Mobile Sink

The End-to-End Delay of three algorithms with different network sizes are shown in Fig. 13. The speed of the mobile sink is at $10 \mathrm{~m} / \mathrm{s}$. According to Fig. 13, the VUGR has lowest end-to-end delay among three algorithms. Moreover, as the network size increases, the VUGR shows good scalability.

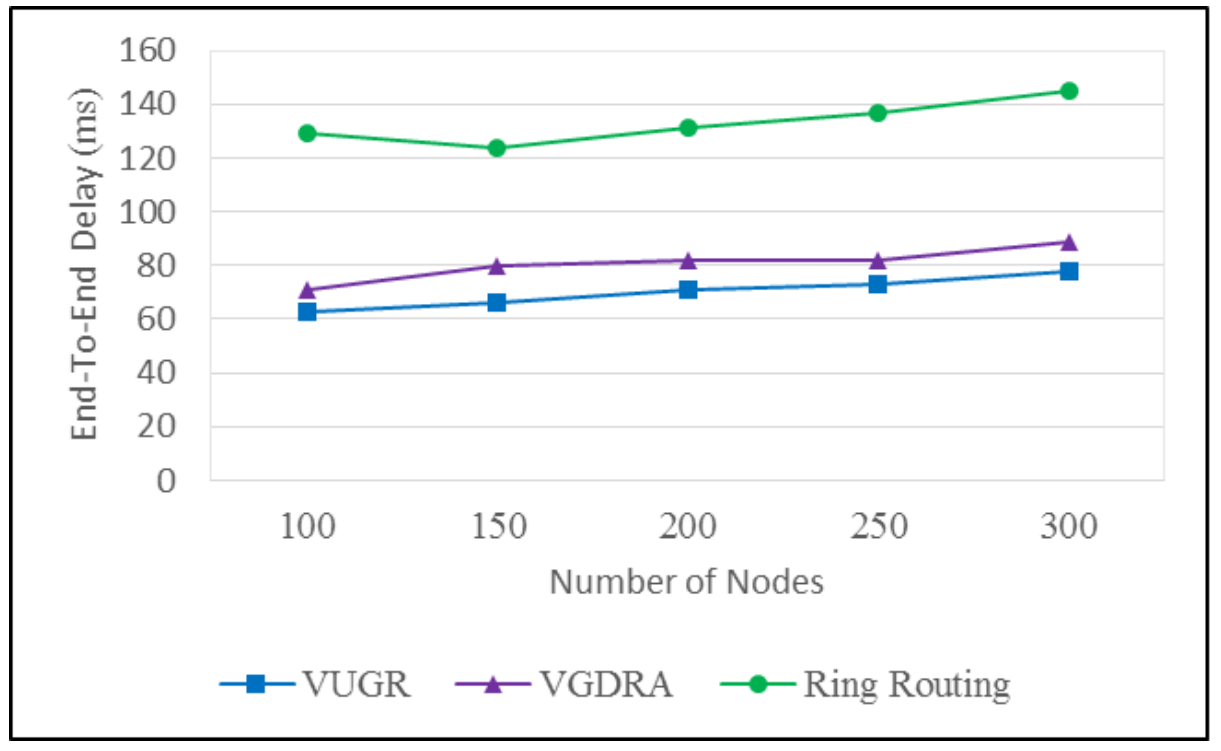

Fig. 13. End-to-end Delay in Different Network Sizes 


\section{$5 \quad$ Conclusion and Future Work}

In this paper, an uneven grid-based routing protocol, called VUGR is presented for energy-efficient scenarios in a practical smart home environment. The VUGR aims to solve the hot-spot problem appearing in grid cells with sparse nodes in a non-uniformly distributed network. When a grid cell along the moving path of the mobile sink depletes battery energy of sensor nodes located in the cell, the proposed VUGR algorithm divides these grid cells owning sparse nodes into smaller cells, which are attached to main grid cells and will not become the data concentrating centre, therefore helps prolonging the network lifetime.

The performance of the VUGR is evaluated via simulated scenarios conducted in NS2.34. According to the experiment results, the VUGR has outperformed better performance on Network Lifetime, Data Delivery Ratio and End-to-end Delay when compared to existing solutions.

At present, the VUGR only considers grid cells with sparse nodes on the edge of the network. In the future, a feasible solution needs to be designed to dynamically adjust all grid cells with sparse nodes for overall energy efficiency of the network.

\section{Acknowledgements}

This work has received funding from the European Union's Horizon 2020 research and innovation programme under the Marie Sklodowska-Curie grant agreement No. 701697.

\section{References}

1. I.F. Akyildiz, W. Su, Y. Sankarasubramaniam and E. Cayirci, "Wireless sensor network: a survey", Computer Networks, vol. 40, no. 8, pp. 393-422, 2002.

2. J. Yick, B. Mukherjee and D. Ghosal, "Wireless sensor network survey", Computer Networks, vol. 52, no. 12, pp. 2292-2330, 2008.

3. M. Chen, S. Gonzalez, A. Vasilakos, H. Cao and C.M. Leung, "Body area networks: a survey", Mobile Networks \& Applications, vol. 16, no. 2, pp.171-193, 2011.

4. M.Z.A. Bhuiyan, G. Wang, J. Cao and J. Wu, "Deploying wireless sensor networks with fault-tolerance for structural health monitoring", IEEE Transactions on Computers, vol. 64, no. 2, pp. 382-395, 2015.

5. N. Grammalidis, E. Cetin, K. Dimitropoulos, F. Tsalakanidou, K. Kose, O. Gunay, B. Kosucu, E.K.D. Torri, S. Tozzi, A. Benazza, F. Chaabane, B. Kosucu and C. Ersoy, "A multi-sensor network for the protection of cultural heritage", Proceeding of 19th European Signal Processing Conference (EUSIPCO2011), Special Session on Signal processing for disaster management and prevention, Aug, 2011.

6. J.N. Al-Karaki and A.E. Kamal, "Routing Techniques in wireless sensor networks: a survey”, IEEE Wireless Communications, vol. 11, no. 6, pp. 6-28, 2004.

7. H. Salarian, K.W. Chin and F. Naghdy, "An energy-efficient mobile-sink path selection strategy for wireless sensor networks", IEEE Transactions on Vehicular Technology, vol. 63, no. 5, pp. 2407-2419, 2014. 
8. M. Abo-Zahhad, S.M. Ahmed, N. Sabor and S. Sasaki, "Mobile sink-based adaptive immune energy-efficient clustering protocol for improving the lifetime and stability period of wireless sensor networks", IEEE Sensors Journal, vol. 15, no. 8, pp. 4576-4586, 2015.

9. C. Konstantopoulos, G. Pantziou, D. Gavalas, A. Mpitziopoulos and B. Mamalis, "A rendezvous-based approach enabling energy-efficient sensory data collection with mobile sinks", IEEE Transactions on Parallel and Distributed Systems, vol. 23, no. 5, pp. 809-817, 2012.

10. C.F. Wang, J.D. Shih, B.H. Pan and T.Y. Wu, "A network lifetime enhancement method for sink relocation and its analysis in wireless sensor networks", IEEE Sensors Journal, vol. 14, no. 6, pp. 1932-1943, 2014.

11. C. Tunca, S. Isik, M.Y. Donmez and C. Ersoy, "Ring Routing: an energy-efficient routing protocol for wireless sensor networks with a mobile sink", IEEE Transactions on Mobile Computing, vol. 14, no. 9, pp. 1947-1960, 2015.

12. C. Tunca, S. Isik, M.Y. Donmez and C. Ersoy, "Distributed mobile sink routing for wireless sensor networks: a survey", IEEE Communications Survey \& Tutorials, vol. 16, no. 2, pp. 877-897, 2014.

13. A.W. Khan, A.H. Abdullah, M.A. Razzaque and J.I. Bangash, "VGDRA: a virtual gridbased dynamic routes adjustment scheme for mobile sink-based wireless sensor networks", IEEE Sensors Journal, vol. 15, no. 1, pp. 526-534, 2015.

14. E.B. Hamida and G. Chelius, "Strategies for data dissemination to mobile sinks in wireless sensor networks", IEEE Wireness Communications, vol. 15, no. 6, pp.31-37, 2008.

15. M. Zhao, Y. Yang and C. Wang, "Mobile data gathering with load balanced clustering and dual data uploading in wireless sensor networks", IEEE Transactions on Mobile Computing, vol. 14, no. 4, pp. 770-785, 2015.

16. H. Luo, F. Ye, J. Cheng, S. Lu and L. Zhang, "TTDD: two-tier data dissemination in largescale wireless sensor networks", Wireless Networks, vol. 11, no. 1-2, pp. 161-175, 2005

17. B. Prince, P. Kumar, M. P. Singh, and J. P. Singh, "An energy efficient uneven grid clustering based routing protocol for Wireless Sensor Networks", Proceedings of 2016 International Conference on Wireless Communications, Signal Processing and Networking (WiSPNET), pp. 1580-1584, Mar, 2016.

18. E.B. Hamida and G. Chelius, "A line-based data dissemination protocol for wireless sensor networks with mobile sink", Proceedings of 2008 IEEE International Conference on Communications, pp. 2201-2205. May, 2008.

19. H.S. Mo, E. Lee, S. Park and S.-H. Kim, "Virtual line-based data dissemination for mobile sink groups in wireless sensor networks", IEEE Communication Letters, vol. 17, no. 9, pp. 1864-1867, 2013.

20. J.H. Shin, J. Kim, K. Park and D. Park, "Railroad: virtual infrastructure for data dissemination in wireless sensor networks", Proceedings of the 2nd ACM International Workshop on Performance Evaluation of Wireless Ad Hoc, Sensor, and Ubiquitous Networks, pp. 168173, Oct, 2005.

21. Z. Mir and Y.-B. Ko, "A quadtree-based hierarchical data dissemination for mobile sensor networks", Telecommunication Systems, vol. 36, pp. 117-128, 2007.

22. C.J. Lin, P.L. Chou and C.-F. Chou, "HCDD: hierarchical cluster-based data dissemination in wireless sensor networks", Proceedings of the 2006 International Conference on Wireless Communications and Mobile Computing, Vancouver, pp. 1189-1194, Aug, 2006.

23. W.B. Heinzelman, A.P. Chandrakasan and H. Balakrishnan, "An application-specific protocol architecture for wireless microsensor networks", IEEE Transactions on Wireless Communications, vol. 1, no. 4, pp. 660-670, 2002. 